\title{
Wavelet Analysis of Bifurcation in a Competition Model
}

\author{
Carlo Cattani and Ivana Bochicchio \\ University of Salerno, \\ Via Ponte Don Melillo, 84084 Fisciano (SA), Italy \\ ccattani@unisa.it, ibochicchio@unisa.it
}

\begin{abstract}
A nonlinear dynamical system which describes two interacting and competing populations (tumor and immune cells) is studied through the analysis of the wavelet coefficients. The wavelet coefficients (also called detail coefficients) are able to reproduce the behaviour of the function, and, being sensible to local changes, are strictly related to the differentiable properties of the function, which cannot be easily derived from the numerical interpolation. So the main features of the dynamical system will be given in terms of detail coefficients that are more adapted to the description of a nonlinear problem.
\end{abstract}

\section{Introduction}

In this paper we consider the nonlinear dynamical system

$$
\left\{\begin{array}{l}
\frac{d x}{d \tau}=\alpha_{1} x-\left[1-\gamma_{0} e^{-2(1+\epsilon) \beta_{4} \tau}\right] x y, \\
\frac{d y}{d \tau}=-\beta_{1} x y-\beta_{2} y+\beta_{3} x+\beta_{4}-\beta_{5} y x^{2}
\end{array}\right.
$$

which represents the competition between two cell populations 46.

The parameters are such that

$$
\left\{\begin{array}{l}
0<\alpha_{1} \\
\left|\beta_{1}\right| \leq 1,0<\beta_{2},\left|\beta_{3}\right| \leq 1,0 \leq \beta_{4} \leq 1 \\
0 \leq \gamma_{0} \leq 1,|\varepsilon|<1
\end{array}\right.
$$

In this system, which is a generalization of the Lotka-Volterra model, the unknown quantity $x(t)$ represents the numerical density of tumor cells, while $y(t)$ is the numerical density of lymphocyte population, under conditions $x(t)>0$ and $y(t)>0[6]$. Moreover 
1. $\alpha_{1}$ is the rate of growth of the tumor population

2. $\beta_{1}$ is the aggressive rate of tumor cells

3. $\beta_{2}$ is the stimulatory effect of the tumor cells on immune cells

4. $\beta_{3}, \beta_{4}$ are, in the average, the immune system response

5. $\beta_{5}$ the tumor malignancy

6. $\varepsilon$ represents the relative velocity of encounter rates of interacting populations.

Finally $\gamma_{0}$ is a parameter related to the ability of recognition of the competing population by the immune system. In particular $\varepsilon$ and $\gamma_{0}$ are coupling the macroscopic with microscopic system 4. Small values of $\gamma_{0}$, according to [4, correspond to the maximum learning, i.e. full recognition of the competing population, whereas $\gamma_{0}=1$ correspond to the minimum learning; the competing population is not recognized by the immune system.

We investigate the dynamics of this system through the analysis of the wavelet coefficients which give the possibility to focus on singularities, local high frequencies variation, irregular structure and transient phenomena (see also [2]). A similar analysis was done in some previous papers 23 where we studied the Van Der Pol equation with and without damping (pointing out stable and stable solutions), where we observed that if the dynamical system is strongly nonlinear, the detail coefficients show significant jumps. Wavelet coefficients strongly depend on local changes, so that when the dynamical system becomes unstable (or chaotic) many effects appear [8]9: the amplitude of the detail coefficients grows, the detail coefficients gather around some peaks, showing also some randomness distribution.

The aim of this paper is to investigate these featuring properties of wavelet coefficients for the above system of equations and to focus on what kind of wavelet coefficients give more precise information about the behavior of the studied dynamical system.

The paper is organized as follows: in Sect. 2 some preliminary definitions about Haar wavelets and short Haar wavelet transform [1 are given. The Lotka-Volterra model is introduced in Sect. 3, where we discuss the solutions of the non linear system through a wavelet analysis.

\section{Short Haar Wavelet Transform}

The Haar scaling function $\varphi(t)$ is the characteristic function on $[0,1]$. By translation and dilation we get the family of functions defined in $[0,1]$

$$
\left\{\begin{array}{l}
\varphi_{k}^{n}(t) \equiv 2^{n / 2} \varphi\left(2^{n} t-k\right), \quad\left(0 \leq n, 0 \leq k \leq 2^{n}-1\right), \\
\varphi\left(2^{n} t-k\right)=\left\{\begin{array}{l}
1, t \in \Omega_{k}^{n} \\
0, t \notin \Omega_{k}^{n} .
\end{array}\right.
\end{array}\right.
$$


The Haar wavelet family $\left\{\psi_{k}^{n}(t)\right\}$ is the orthonormal basis for the $L^{2}([0,1])$ functions [5]:

$$
\left\{\begin{array}{l}
\psi_{k}^{n}(t) \equiv 2^{n / 2} \psi\left(2^{n} t-k\right), \quad\left\|\psi_{k}^{n}(t)\right\|_{L^{2}}=1, \\
\psi\left(2^{n} t-k\right) \equiv \begin{cases}-1, & t \in\left[\frac{k}{2^{n}}, \frac{k+1 / 2}{2^{n}}\right), \\
1, & t \in\left[\frac{k+1 / 2}{2^{n}}, \frac{k+1}{2^{n}}\right), \quad\left(0 \leq n, 0 \leq k \leq 2^{n}-1\right), \\
0, & \text { elsewhere } .\end{cases}
\end{array}\right.
$$

Without loss of generality, we restrict ourselves to $0 \leq n, 0 \leq k \leq 2^{n}-1 \Longrightarrow$ $\Omega_{k}^{n} \subseteq[0,1]$. Let $\boldsymbol{Y} \equiv\left\{Y_{i}\right\},\left(i=0, \ldots, 2^{M}-1,2^{M}=N<\infty, M \in \mathbb{N}\right)$, be a real and square summable time-series $Y \in \mathbb{K}^{N} \subset \ell^{2}$ (where $\mathbb{K}$ is a real field); $t_{i}=i /\left(2^{M}-1\right)$, is the regular equispaced grid of dyadic points on the interval restricted, for convenience and without restriction, to $\Omega=[0,1]$.

Let the set $Y=\left\{Y_{i}\right\}$ of $N$ data be segmented into $\sigma$ segments (in general) of different length. Each segment $\boldsymbol{Y}^{s}, s=0, \ldots, \sigma-1$ is made of $p_{s}=2^{m_{s}}$, $\left(\sum_{s} p_{s}=N\right)$, data:

$$
\boldsymbol{Y}=\left\{Y_{i}\right\}_{i=0, \ldots, N-1}=\bigoplus_{s=0}^{\sigma-1}\left\{\boldsymbol{Y}^{s}\right\}, \quad \boldsymbol{Y}^{s} \equiv\left\{Y_{s p_{s}}, Y_{s p_{s}+1}, \ldots, Y_{s p_{s}+p_{s}-1}\right\}
$$

being, in general, $p_{s} \neq p_{r}$. The short discrete Haar wavelet transform of $\boldsymbol{Y}$ is (see [1]) $\mathrm{W}^{p_{s}, \sigma} \boldsymbol{Y}$, being explicitly $\left(2^{m_{s}}=p_{s}, \sum_{s=0}^{\sigma-1} p_{s}=N\right)$

$$
\left\{\begin{array}{l}
\mathrm{W}^{p_{s}, \sigma} \equiv \bigoplus_{s=0}^{\sigma-1} \mathrm{~W}_{s}^{p}, \boldsymbol{Y}=\bigoplus_{s=0}^{\sigma-1} \boldsymbol{Y}^{s}, \\
\mathrm{~W}^{p_{s}, \sigma} \boldsymbol{Y} \\
\mathrm{W}^{2^{m_{s}}} \boldsymbol{Y}^{s} \\
\left.\bigoplus_{s=0}^{\sigma-1} \mathrm{~W}^{p_{s}}\right) \boldsymbol{Y}=\left(\bigoplus_{s=0}^{\sigma-1} \mathrm{~W}^{p_{s}} \boldsymbol{Y}^{s}\right),
\end{array}\right.
$$

Where the discrete Haar wavelet transform is the operator $\mathrm{W}^{N}: \mathbb{K}^{N} \subset \ell^{2} \rightarrow$ $\mathbb{K}^{N} \subset \ell^{2}$ which maps the vector $\boldsymbol{Y}$ into the vector of the wavelet coefficients $\left\{\alpha, \beta_{k}^{n}\right\}$ :

$$
\mathrm{W}^{N} \boldsymbol{Y}=\left\{\alpha, \beta_{0}^{0}, \ldots, \beta_{2^{M-1}-1}^{M-1}\right\}, \quad \boldsymbol{Y}=\left\{Y_{0}, Y_{1}, \ldots, Y_{N-1}\right\} .
$$

There follows that, the matrix of the wavelet transform is expressed as a direct sum of lower order matrices so that the short transform is a sparse matrix [1]. We want to emphasize that when the short wavelet transform maps short interval values into a few set of wavelet coefficients, it can be considered as a first order approximation. Thus giving information about the linear behavior. However, 
since the wavelet transform maps the original signal into uncorrelated sequences [7, the short wavelet transform describes for each sequence of detail coefficients its local behavior. When $p_{s}=p=N, \sigma=1$, the above coincides with the ordinary wavelet transform. We assume, in the following, $p_{s}=p=N / \sigma, s=$ $0, \ldots, \sigma-1,(\sigma>1)$.

\section{System of Competition}

Let us consider the competition model (11); by fixing some parameters

$$
\left\{\begin{array}{l}
\frac{d x}{d \tau}=x-\left[1-e^{-2(1+0.1) 0.2 \tau}\right] x y, \\
\frac{d y}{d \tau}=-0.1 y+x+0.2-\beta_{5} y x^{2},
\end{array}\right.
$$

we obtain the numerical solution as in Fig. 1. where we take, as initial conditions, an initial high numerical density of tumor cells $x(0)=5$ and we neglect the initial number of lymphocytes $y(0)=0$. The other parameters are taken as $\gamma_{0}=1, \varepsilon=0.1$ and, according to (5), we simulate a competition where the rate of growth of the tumor population is little $\left(\alpha_{1}=1\right)$, the aggressive rate of tumor cells is neglectable $\left(\beta_{1}=0\right)$, the stimulatory effect of the tumor cells on immune cells is weak $\left(\beta_{2}=0.1\right)$, the immune system response is in the average $\beta_{3}=1, \beta_{4}=0.2$. Of course with higher values of $\beta_{4}$ we will have a stronger immune system (weaker for smaller values of $\beta_{4}$ ).

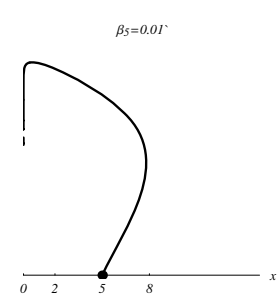

$\beta_{5}=0.05$

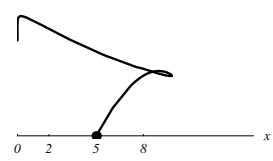

$\beta_{S}=0.04$

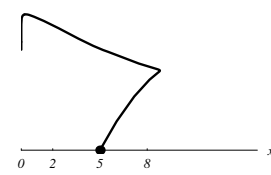

$\beta_{5}=0.06$

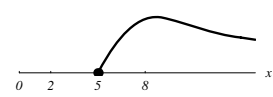

Fig. 1. Numerical solution of system (1) with parameters $\alpha_{1}=1, \beta_{1}=0, \beta_{2}=$ $0.1, \beta_{3}=1, \beta_{4}=0.2, \gamma_{0}=1, \varepsilon=0.1$, and initial conditions $x(0)=5, y(0)=0$, in correspondence of different values of $\beta_{5}$ 

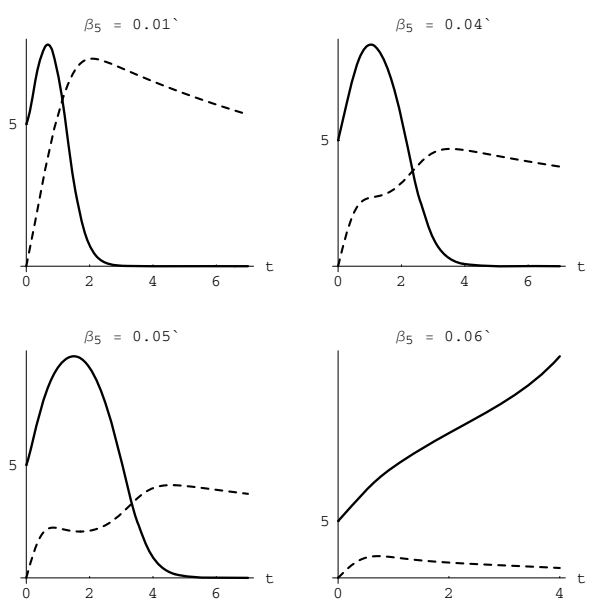

Fig. 2. Numerical solution (plain $x(t)$, dashed $y(t)$ ) of system (1) with parameters $\alpha_{1}=1, \beta_{1}=0, \beta_{2}=0.1, \beta_{3}=1, \beta_{4}=0.2, \gamma_{0}=1, \varepsilon=0.1$, and initial conditions $x(0)=5, y(0)=0$, in correspondence of different values of $\beta_{5}$

It can be observed (see Fig. 1 and Fig. 2) that with small values of the tumor malignancy $\beta_{5}$, the lymphocyte population $y(t)$ grows, whereas tumor cells $x(t)$ decrease. When $\beta_{5}>0.05$ the number of lymphocytes reach a maximum value and then goes to zero while the tumor cell population grows. It should be noticed that $\beta_{5}=0.05$ represents a bifurcation point for the model with loss of uniqueness of the differential system, because the curve is not simply connected (for the presence of a knot). When the number of tumor cells tends to zero the number of lymphocytes decreases to a particular value, called the sentinel value. The dynamics of equation (5) has been simulated by using the Runge Kutta 4 -th order method, with the accuracy $10^{-6}$. We obtain as a numerical solution $(0<t \leq 6)$, in correspondence of the values of the parameter $\alpha_{1}=1, \beta_{1}=0, \beta_{2}=0.1, \beta_{3}=1, \beta_{4}=0.2, \gamma_{0}=1, \varepsilon=0.1$, four sequences (in correspondence of $\beta_{5}=0.01, \beta_{5}=0.04, \beta_{5}=0.05, \beta_{5}=0.06$ ) of $2^{9}=512$ values $\boldsymbol{Y}=\left\{Y_{0}, Y_{1}, \ldots, Y_{N-1}\right\}$, with $N=512$ and $M=9$. Moreover, using the short Haar wavelet transform, with $p_{s}=p=4$, we compare the wavelet coefficients of the two time-series, near the bifurcation value of $\beta_{5}$, i.e. $\beta_{5}=0.05$ and $\beta_{5}=0.06$ computed numerically (Fig. 3. 4).

\section{Critical Analysis}

The importance of the wavelet analysis is mainly based on the information content of the detail coefficients. It can be seen (from Fig. 3and 4) that only a small set of detail coefficients, namely $\beta_{0}^{0}, \beta_{0}^{1}, \beta_{1}^{1}$, is already enough to have a good information about the dynamical system, in any case better than the numerical evaluation (Fig. 34 on top). In fact, the detail coefficients show some local 

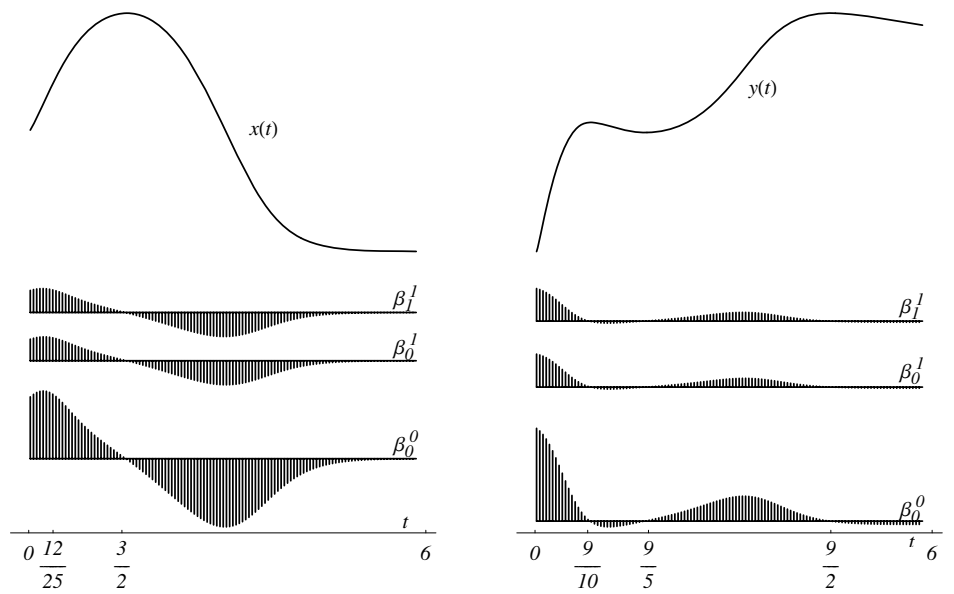

Fig. 3. Numerical solution and wavelet coefficient of 4-parameters of short Haar transform of the numerical solution $x(t)$ (left) and $y(t)$ (right) of system (1) with parameters $\alpha_{1}=1, \beta_{1}=0, \beta_{2}=0.1, \beta_{3}=1, \beta_{4}=0.2, \gamma_{0}=1, \varepsilon=0.1$, and initial conditions $x(0)=5, y(0)=0$, in correspondence of $\beta_{5}=0.05$
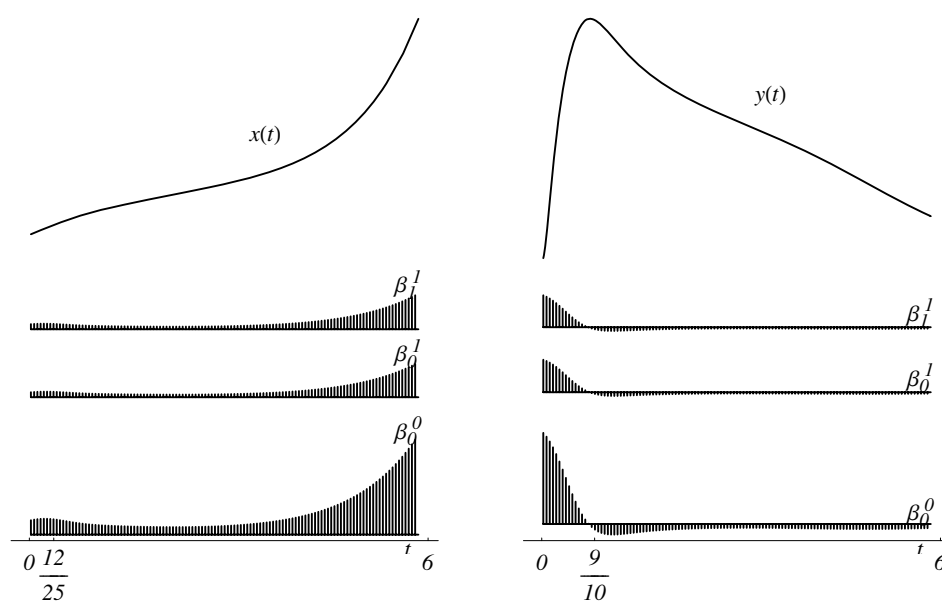

Fig. 4. Numerical solution and wavelet coefficient of 4-parameters of short Haar transform of the numerical solution $x(t)$ (left) and $y(t)$ (right) of system (1) with parameters $\alpha_{1}=1, \beta_{1}=0, \beta_{2}=0.1, \beta_{3}=1, \beta_{4}=0.2, \gamma_{0}=1, \varepsilon=0.1$, and initial conditions $x(0)=5, y(0)=0$, in correspondence of $\beta_{5}=0.06$

maxima and changes which are hidden in the continuous interpolation of the numerical integration. Each detail coefficient is able, at each scale but mostly at the lower scale $n=0, \beta_{0}^{0}$, to reproduce the behaviour of the function, but they are very sensible to local changes and therefore they can easily describe the intervals where the function is monotonic. Moreover, being sensible to local changes they 
are strictly related to the differentiable properties of the function, which cannot be easily derived from the numerical interpolation of the function. In particular, we can see that they can focus exactly on local maxima of the function, and on a number of changes higher than the numerical functions $x(t)$ e $y(t)$. Therefore the detail coefficients are more adapted to the description of a nonlinear problem but also show a inflexion in the initial growth which is invisible in the numerical solution. These time spots, where the the detail coefficients are zero, or where the detail coefficients have some local maxima (minima) are reported in Fig. 34, and tell us the inversion (inflexion) in the population growth. The positive values of the detail coefficients describe the local growth, the negative values the decreasing of the function. Local maxima (minima) of the detail coefficients define some inflexion which enable us to predict if the phenomenon will increase in time or decrease.

\section{References}

1. Cattani, C.: Haar Wavelet based Technique for Sharp Jumps Classification. Mathematical Computer Modelling 39 (2004) 255-279.

2. Cattani, C., Bochicchio, I.: Wavelet Analysis of Chaotic Systems. Journal of Interdisciplinary Mathematics Vol. 9 No. 3 (2006) 445-458.

3. Cattani, C., Bochicchio, I.: Clustering Dynamical System by Wavelet. Proceeding of the International Conference "Inverse Problem and Applications" Birsk, Russia (2006) 149-159

4. Cattani, C., Ciancio, A.: Hybrid Two Scales Mathematical Tools for Active Particles Modelling Complex System with Learning Hiding Dynamics. Math. Mod. Meth. Appl. Sci. Vol. 2 No 17 (2007).

5. Daubechies, I.: 'Ten lectures on wavelets. CBMS-NSF Regional Conference Series in Applied Mathematics, SIAM, Philadelphia (1992).

6. D'Onofrio, A.: A general framework for modeling tumor-immune system competition and immunotherapy: Mathematical analysis and biomedical inference. Physica D 208 (2005) 220-235.

7. Percival, D. B., Walden, A. T.: Wavelet Methods for Time Series Analysis. Cambridge University Press, Cambridge (2000).

8. Toma, C.: An Extension of the Notion of Observability at Filtering and Sampling Devices. Proceedings of the International Symposium on Signals, Circuits and Systems Iasi SCS, Romania (2001) 233-236.

9. Toma, G.: Practical Test Functions Generated by Computer Algorithms. Lecture Notes Computer Science 3482 (2005) 576-585. 